\title{
SOME CHARACTERIZATIONS OF DEVELOPABLE SPACES
}

\author{
HARALD BRANDENBURG
}

\begin{abstract}
Two characterizations of developable spaces are proved which may be viewed as analogues, for developable spaces, of the Nagata-Smirnov metrization theorem or of the "double sequence metrization theorem" of Nagata respectively.
\end{abstract}

1. Introduction. A sequence $\left(Q_{n}\right)_{n \in N}$ of open covers of a topological space $X$ is called a development of $X$, if for each $x \in X$ the collection $\left\{\operatorname{St}\left(x, \mathbb{Q}_{n}\right) \mid n \in \mathbf{N}\right\}$ is a neighbourhood base of $x$, where $\operatorname{St}\left(x, \mathbb{Q}_{n}\right)=\cup\left\{A \mid A \in \mathbb{Q}_{n}, x \in A\right\}$. Following $\mathrm{R}$. H. Bing [2] a topological space is called developable if it has a development. This class of spaces has turned out to be one of the most natural and useful generalizations of metrizable spaces. In fact, several important characterizations of metrizable topological spaces in terms of developments having certain additional properties have been formulated by Alexandroff-Urysohn, Moore, Jones and Arhangel'skii (see [7] for a survey of metrization theory). Many other results in metrization theory make use of Bing's fundamental theorem that a $T_{1}$-space is metrizable if and only if it is collectionwise normal and developable [2].

During the last twenty years a great variety of characterizations of developable spaces involving many different concepts appeared in the literature. These results are too numerous to cite here. In particular, for regular or completely regular $T_{1}$-spaces there are several interesting factorizations of developability in terms of $G_{\delta}$-diagonal concepts, (generalizations of) $p$-spaces, $\theta$-refinability, (generalizations of) semistratifiability, quasi-developability or symmetrizability. Probably the most interesting among the internal characterizations of arbitrary developable spaces is the basic theorem of $\mathrm{H}$. H. Wicke and J. M. Worrell, Jr. which expresses developability essentially as a combination of a base property (i.e. base of countable order) and a weak covering property (i.e. $\theta$-refinability) [10].

Every characterization of developable spaces yields a (not always significant) metrization theorem by simply adding collectionwise normality. On the other hand one may ask whether some basic metrization theorems have counterparts in the theory of developable spaces. The above mentioned theorem of Wicke-Worrell, Jr., for instance, generalizes a metrization theorem of A. Arhangel'skii [1] which itself is an improvement of the Alexandroff-Urysohn metrization theorem. It is the aim of this paper to provide two new developability theorems which may be viewed as analogues of the Nagata-Smirnov theorem or of the "double sequence theorem" of

Received by the editors April 2, 1979 and, in revised form, June 27, 1979.

AMS (MOS) subject classifications (1970). Primary 54E30.

Key words and phrases. Developable space, dissection, dissectable space. 
J.-I. Nagata [9] respectively. For this purpose we first introduce a new covering property which generalizes paracompactness in perfect spaces. It is shown that every developable space has this property. As a consequence of the main result we obtain a characterization of second countable developable spaces which generalizes the classical Urysohn metrization theorem.

2. Results. In order to prove a characterization of developability similar to the Nagata-Smirnov theorem it is necessary to find a suitable generalization of locally finite open collections. Since metrizable as well as developable spaces are perfect (i.e. all open sets are $F_{\sigma}$-sets), even an appropriate generalization of locally finite collections consisting of open $F_{\sigma}$-sets would suffice. We introduce the following notion.

2.1. Definition. Let $X$ be a topological space and $\mathbb{Q}=(A(i))_{i \in I}$ be a collection of subsets of $X$. A function $D: I \times \mathbf{N} \rightarrow \mathscr{P}(X)$ is called a dissection of $\mathscr{Q}$ if it satisfies the following conditions:

(i) $A(i)=\cup\{D(i, n) \mid n \in \mathrm{N}\}$ for each $i \in I$.

(ii) $T(D, n)=\cup\{D(i, n) \mid i \in I\}$ is closed for each $n \in \mathbf{N}$.

(iii) For each $n \in \mathbf{N}$ and for each $x \in T(D, n)$ the set $\cap\{A(i) \mid i \in I, x \in$ $D(i, n)\} \cap X \backslash \cup\{D(i, n) \mid i \in I, x \notin D(i, n)\}$ is a neighbourhood of $x$.

The collection $\mathscr{Q}$ is called dissectable if there exists a dissection of $\mathscr{Q}$. The space $X$ is called dissectable if every open cover of $X$ has an open refinement which is dissectable.

2.2. LemMa. Every locally finite collection of open $F_{\sigma}$-subsets of a topological space is dissectable.

Proof. Let $X$ be a topological space and let $\mathbb{Q}=(A(i))_{i \in I}$ be a locally finite collection of open $F_{\sigma}$-subsets of $X$. For each $i \in I$ there exists a sequence $(A(i, n))_{n \in \mathbf{N}}$ of closed subsets of $X$ such that $A(i)=\cup\{A(i, n) \mid n \in \mathbf{N}\}$. Define $D(i, n)=A(i, n)$ for each $(i, n) \in I \times \mathbf{N}$. It is easy to see that $D: I \times \mathbf{N} \rightarrow \mathscr{P}(X)$ is a dissection of $\mathbb{Q}$.

By virtue of Lemma 2.2 every perfect paracompact space is dissectable. Moreover it can be shown that every fully normal space (and therefore every paracompact Hausdorff space) is dissectable. Every dissectable space is subparacompact, but there are subparacompact spaces which are not dissectable. A detailed study of dissectable spaces containing these results will appear elsewhere. The only fact we need here is that every developable space is dissectable, which is an immediate consequence of the following theorem.

\subsection{THEOREM. Every open cover of a developable space is dissectable.}

Proof. Let $X$ be a developable space and let $\mathscr{Q}=(A(i))_{i \in I}$ be an open cover of $X$. There exists a development $\left(\mathscr{B}_{n}\right)_{n \in \mathrm{N}}$ of $X$ such that for every point $x \in X$ and for every neighbourhood $U$ of $x$ there is a positive integer $n$ such that there is only one set in $\mathscr{B}_{n}$ containing $x$ and it is a subset of $U$ [6]. For each $n \in \mathbf{N}$ denote by $H(n)$ the set of all points of $X$ which are contained in exactly one member of $\mathscr{B}_{n}$. For each $x \in H(n)$ define $A(x, n)=X \backslash \cup\left\{B \mid B \in \mathscr{B}_{n}, x \notin B\right\}$. Obviously $x \in$ 
$A(x, n) \subset B(x, n)$, where $B(x, n)$ denotes the element of $\mathscr{B}_{n}$ which contains $x$. If $A(i, n)=\bigcup\{A(x, n) \mid x \in H(n), B(x, n) \subset A(i)\}$ for each $(i, n) \in I \times \mathbf{N}$, we claim that $D: I \times \mathbf{N} \rightarrow \mathscr{P}(X)$ defined by $D(i, n)=A(i, n)$ is a dissection of $\mathbb{Q}$.

To prove this assertion we first show that for each $n \in \mathbf{N}$ the collection $\mathbb{Q}_{n}=\{A(x, n) \mid x \in H(n)\}$ is discrete. Consider a fixed $n \in \mathbf{N}$ and an arbitrary point $z \in X$. There is a $B(z) \in \mathscr{B}_{n}$ such that $z \in B(z)$. If there is a point $x \in H(n)$ such that $A(x, n) \cap B(z) \neq \varnothing$, then $B(z)=B(x, n)$. Hence there is at most one element of $\mathbb{Q}_{n}$ which intersects $B(z)$, i.e. $\mathbb{Q}_{n}$ is discrete, and therefore closure-preserving.

Now we show that the mapping $D$ satisfies the conditions (i)-(iii) of 2.1. Since for each $x \in A(i)$ there is a positive integer $n(x)$ such that $x \in A(x, n(x)) \subset$ $B(x, n(x)) \subset A(i)$, it follows that $A(i)=\bigcup\{A(i, n) \mid n \in \mathbf{N}\}$ for each $i \in I$. That $T(D, n)=\cup\{A(i, n) \mid i \in I\}$ is a closed set for each $n \in \mathbf{N}$ is an immediate consequence of the fact that $\mathcal{Q}_{n}$ is a closure-preserving collection of closed sets. Therefore it remains to prove that for each $n \in \mathbf{N}$ and for each $z \in T(D, n)$ there exists an open set $O(z, n)$ such that $z \in O(z, n) \subset \cap\{A(i) \mid i \in I, z \in A(i, n)\}$ and $O(z, n) \subset X \backslash \cup\{A(i, n) \mid i \in I, z \notin A(i, n)\}$. Let $n$ and $z$ be fixed. There is an $x_{0} \in H(n)$ such that $z \in A\left(x_{0}, n\right)$. We claim that $O(z, n)=B\left(x_{0}, n\right)$ has the desired properties.

For the proof of this statement consider an $i \in I$ such that $z \in A(i, n)$. There exists a $y \in H(n)$ such that $z \in A(y, n) \subset B(y, n) \subset A(i)$. Since $\mathbb{Q}_{n}$ is discrete, it follows that $A\left(x_{0}, n\right)=A(y, n)$, which implies that $O(z, n) \subset A(i)$. This proves that $O(z, n) \subset \cap\{A(i) \mid i \in I, z \in A(i, n)\}$. Now suppose that there is an $i \in I$ such that $z \notin A(i, n)$ and $O(z, n) \cap A(i, n) \neq \varnothing$. Then there exists a point $u \in$ $O(z, n) \cap A(i, n)$ and a point $v \in H(n)$ such that $u \in A(v, n) \subset B(v, n) \subset A(i)$. Consequently $B(v, n)=B\left(x_{0}, n\right)$, for otherwise we would have $u \in O(z, n)=$ $B\left(x_{0}, n\right) \subset \cup\left\{B \mid B \in \Re_{n}, v \notin B\right\}=X \backslash A(v, n)$, which is impossible. Since $B(v, n)=B\left(x_{0}, n\right)$ implies $A(v, n)=A\left(x_{0}, n\right)$, it follows that $z \in A(v, n) \subset$ $B(v, n) \subset A(i)$, i.e. $z \in A(i, n)$-a contradiction! Therefore

$$
O(z, n) \subset X \backslash \bigcup\{A(i, n) \mid i \in I, z \notin A(i, n)\},
$$

which completes the proof.

We call a collection $Q$ of subsets of a topological space $X \boldsymbol{\sigma}$-dissectable if $\mathcal{Q}$ is a countable union of dissectable collections of subsets of $X$. Our first characterization of developable spaces is an immediate consequence of the preceding result.

2.4. TheOrem. A topological space is developable if and only if it has a $\sigma$-dissectable open base.

Proof. Let $X$ be a topological space. Suppose that $X$ has a development $\left(\mathbb{Q}_{n}\right)_{n \in N}$. By virtue of $2.3 \mathscr{Q}=\bigcup\left\{Q_{n} \mid n \in \mathbf{N}\right\}$ is a $\sigma$-dissectable open base of $X$. For the proof of the reverse implication assume that $X$ has a $\sigma$-dissectable open base $\mathscr{B}=\bigcup\left\{\mathscr{B}_{n} \mid n \in \mathbf{N}\right\}$, where $\mathscr{B}_{n}=(B(i))_{i \in I(n)}$ for each $n \in \mathbf{N}$. Let $D_{n}: I(n) \times \mathbf{N}$ $\rightarrow \mathscr{P}(X)$ be a dissection of $\mathscr{B}_{n}$. For each pair $n, k$ we construct an open cover $\mathbb{Q}(n, k)$ of $X$ as follows. If $x \in T\left(D_{n}, k\right)=\cup\left\{D_{n}(i, k) \mid i \in I(n)\right\}$ there exists an open set $O(x, n, k)$ such that $x \in O(x, n, k) \subset \bigcap\left\{B(i) \mid i \in I(n), x \in D_{n}(i, k)\right\}$ 
and $O(x, n, k) \subset X \backslash \cup\left\{D_{n}(i, k) \mid i \in I(n), x \notin D_{n}(i, k)\right\}$. We define $\mathcal{Q}(n, k)=$ $\left\{X \backslash T\left(D_{n}, k\right)\right\} \cup\left\{O(x, n, k) \mid x \in T\left(D_{n}, k\right)\right\}$ and claim that $(\mathbb{Q}(n, k))_{(n, k) \in \mathrm{N} \times \mathrm{N}}$ is a development of $X$. To prove this assertion consider an arbitrary point $x \in X$ and a neighbourhood $U$ of $x$. Since $\mathscr{B}$ is a base of $X$, there exists an $n \in \mathbf{N}$ and an $i_{0} \in I(n)$ such that $x \in B\left(i_{0}\right) \subset U$. Furthermore there is a $k \in \mathbf{N}$ such that $x \in D_{n}\left(i_{0}, k\right)$. It remains to prove that $\operatorname{St}(x, \mathbb{Q}(n, k)) \subset U$. Since $\operatorname{St}(x, \mathbb{Q}(n, k))=$ $\cup\left\{O(y, n, k) \mid y \in T\left(D_{n}, k\right), x \in O(y, n, k)\right\}$, it is sufficient to show that for each $y \in T\left(D_{n}, k\right)$ with $x \in O(y, n, k)$ the set $O(y, n, k)$ is contained in $B\left(i_{0}\right)$. Consider a fixed $y \in T\left(D_{n}, k\right)$ such that $x \in O(y, n, k)$. For every $j \in I(n)$ such that $x \in D_{n}(j, k)$ we have $y \in D_{n}(j, k)$, for otherwise we would have $x \in D_{n}(j, k) \subset$ $\cup\left\{D_{n}(i, k) \mid i \in I(n), y \notin D_{n}(i, k)\right\} \subset X \backslash O(y, n, k)$, which is impossible. Hence $O(y, n, k) \subset \cap\left\{B(i) \mid i \in I(n), y \in D_{n}(i, k)\right\} \subset \cap\left\{B(i) \mid i \in I(n), x \in D_{n}(i, k)\right\}$ $\subset B\left(i_{0}\right)$, which completes the proof.

An open base $\mathscr{B}$ for a topological space $X$ is called locally countable if each point $x$ of $X$ has a neighbourhood meeting just countably many members of $\mathscr{B}$. In [5] it was shown that every space $X$ with a locally countable open base also has a $\sigma$-locally finite open base. Therefore every regular $T_{1}$-space with a locally countable base is metrizable [5]. If one drops regularity one obtains the following result.

2.5. Corollary. Every topological space with a locally countable base consisting of open $F_{\sigma}$-sets is developable.

Proof. Let $X$ be a topological space with a locally countable base consisting of open $F_{\sigma}$-sets. It follows from the construction performed in [5] that $X$ has a $\sigma$-locally finite base consisting of open $F_{\sigma}$-sets. By virtue of 2.2 and $2.4 X$ is developable.

The next theorem can be viewed as an analogue, for developable spaces, of Urysohn's metrization theorem. It was first proved in [4] without relying on 2.4.

2.6. THEOREM. For a second countable space $X$ the following statements are equivalent:

(1) $X$ is developable.

(2) $X$ is a subspace of a product of developable spaces.

(3) For every closed set $A \subset X$ and for every $x \in X \backslash A$ there exists a developable space $Y$ and a continuous mapping $f: X \rightarrow Y$ such that $\operatorname{cl} f[A] \cap \operatorname{cl}\{f(x)\}=\varnothing$.

(4) $X$ is a subspace of a product of perfect spaces.

(5) $X$ has a base consisting of open $F_{o}$-sets.

Proof. Obviously (1) implies (2). In [3] it was shown that (2), (3) and (4) are equivalent. In particular, it was shown that $X$ is a subspace of a product of perfect spaces if and only if $X$ has a base $\mathscr{B}$ for the closed sets with the property that for each $B \in \mathscr{B}$ there is a sequence $\left(B_{n}\right)_{n \in \mathrm{N}}$ in $\mathscr{B}$ such that $X \backslash B=\bigcup\left\{B_{n} \mid n \in \mathbf{N}\right\}$. Therefore (4) implies (5). Assuming (5) it is easy to construct a countable base of $X$ consisting of open $F_{o}$-sets. Hence by virtue of 2.2 and $2.4 X$ is developable, which completes the proof.

Let us note that it is possible to derive a proof of the Urysohn metrization 
theorem from 2.6. In fact, if $X$ is a regular $T_{1}$-space with a countable base, then $X$ is paracompact [8]. Since a paracompact Hausdorff space has a base consisting of open $F_{\sigma}$-sets, $X$ is developable by virtue of 2.6 and therefore metrizable [2].

Our second characterization of developable spaces is motivated by J.-I. Nagata's characterization of metrizable spaces [9].

2.7. TheOREM. A topological space $X$ is developable if and only if for each point $x$ of $X$ there exist two sequences $(U(x, n))_{n \in \mathbf{N}}$ and $(V(x, n, k))_{(n, k) \in \mathbf{N} \times \mathbf{N}}$ of neighbourhoods of $x$ such that

(i) $(U(x, n))_{n \in \mathbf{N}}$ is a neighbourhood base of $x$, and for each $n \in \mathbf{N} U(x, n)$ has a decomposition $U(x, n)=\cup\{U(x, n, k) \mid k \in \mathbf{N}\}$ such that

(ii) $y \notin U(x, n, k)$ implies $V(y, n, k) \cap U(x, n, k)=\varnothing$, and

(iii) $y \in U(x, n, k)$ implies $V(y, n, k) \subset U(x, n)$.

Proof. Let $\left(\mathbb{Q}_{n}\right)_{n \in \mathrm{N}}$ be a development of $X$, where $\mathbb{Q}_{n}=(A(i))_{i \in I(n)}$. For each $n \in \mathbf{N}$ there exists a dissection $D_{n}: I(n) \times \mathbf{N} \rightarrow \mathscr{P}(X)$ of $\mathbb{Q}_{n}(2.3)$. For each $x \in X$ and for each $n \in \mathbf{N}$ choose a fixed $i(x, n) \in I(n)$ such that $x \in A(i(x, n))$. We define $U(x, n)=A(i(x, n))$ and $U(x, n, k)=D_{n}(i(x, n), k)$ for each $n, k \in \mathbf{N}$. Furthermore we put

$$
\begin{aligned}
& V(x, n, k)=\bigcap\left\{A(i) \mid i \in I(n), x \in D_{n}(i, k)\right\} \cap X \backslash \\
& \bigcup\left\{D_{n}(i, k) \mid i \in I(n), x \notin D_{n}(i, k)\right\} .
\end{aligned}
$$

It is immediate that the so-defined sequences of neighbourhoods of $x$ satisfy (i)-(iii). For the proof of the reverse implication define $A(x, n, k)=U(x, n) \cap$ $V(x, n, k)$ and $\mathcal{Q}(n, k)=(A(x, n, k))_{x \in X}$ for each pair $n, k \in \mathbf{N}$. Since it is easy to see that $(\mathscr{Q}(n, k))_{(n, k) \in \mathbf{N} \times \mathbf{N}}$ is a development of $X$, the proof is complete.

\section{REFERENCES}

1. A. Arhangel'skii, Some metrization theorems, Uspehi Mat. Nauk 18 (1963), 139-145. (Russian)

2. R. H. Bing, Metrization of topological spaces, Canad. J. Math. 3 (1951), 175-186.

3. H. Brandenburg, On a class of nearness spaces and the epireflective hull of developable topological spaces, Proc. Internat. Sympos. on Topology and its Appl. (Beograd, 1977) (to appear).

4. , On spaces with $a G_{\delta}$-basis (submitted).

5. A. Charlesworth, A note on Urysohn's metrization theorem, Amer. Math. Monthly 83 (1976), 718-720.

6. J. W. Green, Completion and semicompletion of Moore spaces, Pacific J. Math. 57 (1975), 153-165.

7. R. E. Hodel, Some results in metrization theory 1950-1972, Topology Conference Virginia Polytechnic Inst. and State University, Lecture Notes in Math., vol. 375, Springer-Verlag, Berlin and New York, 1974, pp. 120-136.

8. K. Morita, Star-finite coverings and star-finite property, Math. Japon. 1 (1948), 60-68.

9. J.-I. Nagata, A contribution to the theory of metrization, J. Inst. Polytech. Osaka City Univ. Ser. A Math. 8 (1957), 185-192.

10. J. M. Worrell, Jr. and H. H. Wicke, Characterizations of developable topological spaces, Canad. J. Math. 17 (1965), 820-830.

Freie UnVersität Berlin, Institut für Mathematik I, D 1000 Berlin 33, Federal Republic of Germany 